JOURNAL OF SYNCHROTRON RADIATION

ISSN $1600-5775$

Received 24 January 2019

Accepted 20 February 2019

Edited by M. Yamamoto, RIKEN SPring-8 Center, Japan

Keywords: X-ray imaging; biological cells; dose and fluence requirements.

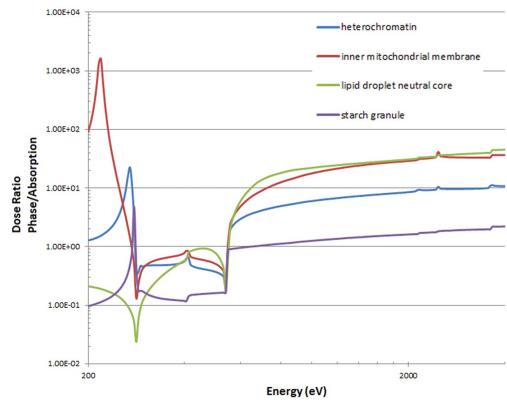

OPEN $\odot$ ACCESS

\section{A comparison of absorption and phase contrast for X-ray imaging of biological cells. Erratum}

\author{
Colin Nave*
}

Diamond Light Source Ltd, Harwell Science and Innovation Campus, Didcot OX11 ODE, UK. *Correspondence e-mail: colin.nave@diamond.ac.uk

An error in the calculation for X-ray absorption imaging has been identified in the paper by Nave (2018) [J. Synchrotron Rad. 25, 1490-1504]. The required fluence and dose in the paper are a factor of ten too low for this mode of imaging.

The author regrets that an error in the calculations of the required fluence (and hence dose) for imaging by absorption was made in the paper by Nave (2018). This occurred because an incorrect number was applied when converting from the required number of photons incident on a pixel to a fluence (photons $\mu \mathrm{m}^{-2}$ ). The fluences and doses shown in Figs. 3 and 6 should all be increased by a factor of ten. As the error only occurred in the calculation for imaging by absorption, the dose ratios in Fig. 7 should be decreased by a factor of ten. These ratios are discussed in the text where the following corrections should be made:

Section 5.4: 'For some components, much higher doses (e.g. a factor of 10-45 times more) are required for phase contrast imaging at the higher energies.'

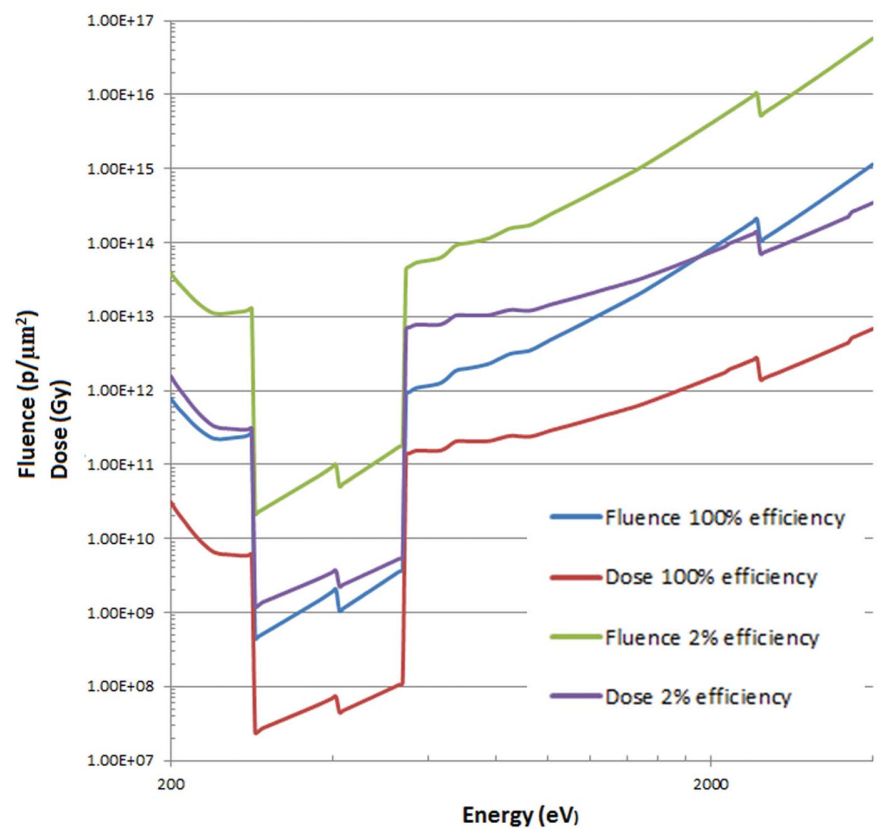

Figure 3

Fluence and dose for protein in water with absorption contrast. Rose criteria 5 with the dose distributed over a model cell $(70 \%$ water). The fluence and dose at $2 \%$ efficiency follows the zone plate efficiency adopted by Huang et al. (2009). 

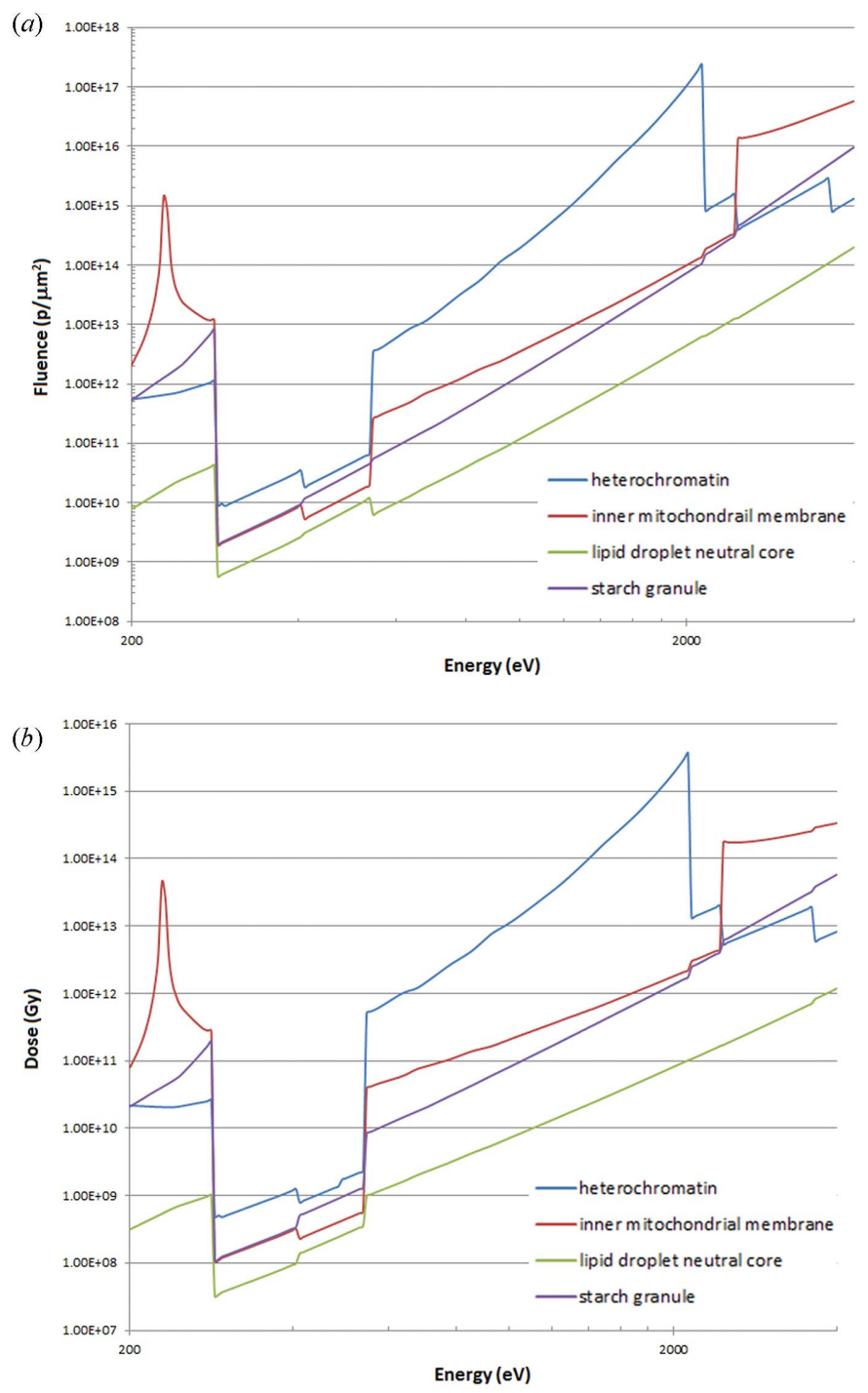

Figure 6

(a) Fluence requirements (absorption contrast, $10 \mathrm{~nm}$ resolution) for the four cellular components following the calculations for protein illustrated in Fig. 3, 100\% efficiency. (b) Dose requirements following the calculations in Fig. 3, 100\% efficiency.

Section 6, fourth paragraph: 'The dose requirements for phase contrast at $4000 \mathrm{eV}$ are between a factor of 2.2 (starch

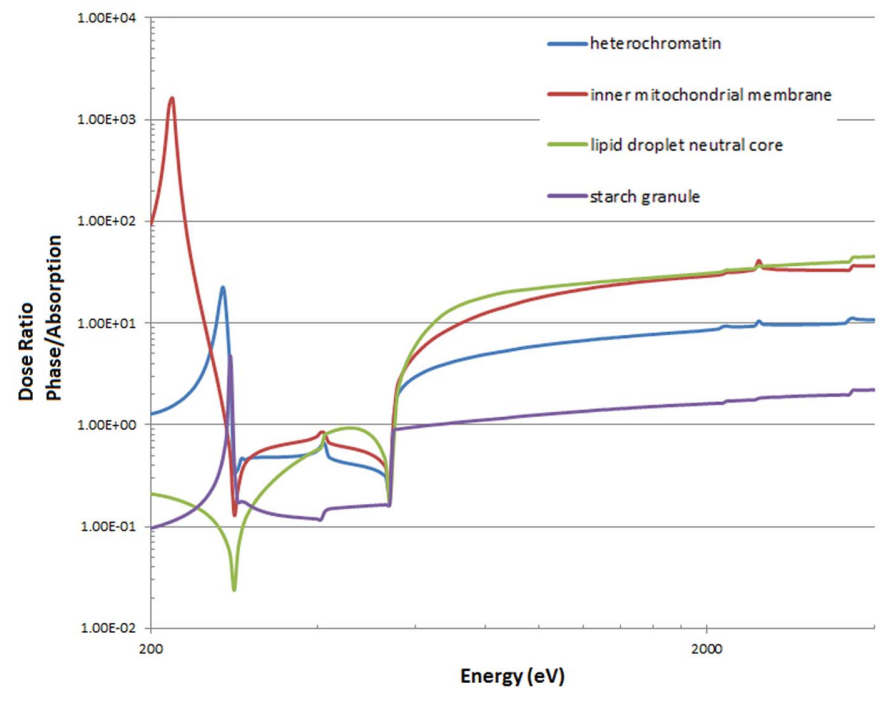

Figure 7

Comparison of the dose for phase contrast with absorption contrast at $520 \mathrm{eV}$. Obtained by dividing the values in Fig. $5(b)$ with the value at $520 \mathrm{eV}$ in Fig. 6(b).

granule) and 45 (lipid droplet) higher than absorption contrast at $520 \mathrm{eV}$ (Fig. 7). Mitochondrial membranes in absorption contrast at $522 \mathrm{eV}$ would require a dose of $5.2 \times 10^{8} \mathrm{~Gy}$ for $10 \mathrm{~nm}$ resolution whereas a dose of $1.5 \times 10^{10}$ Gy would be required in phase contrast at $2000 \mathrm{eV}$.'

The benefits of operating in the water window for thin specimens are still present although to a lesser extent than given in the paper. The error was identified when comparing the calculations with those of Schneider (1998) in which optimized phase contrast (exploiting amplitude and phase contrast) in the water window gave the lowest required dose for thin specimens. The corrections given above are consistent with this conclusion.

\section{References}

Huang, X., Miao, H., Steinbrener, J., Nelson, J., Shapiro, D., Stewart, A., Turner, J. \& Jacobsen, C. (2009). Opt. Express, 17, 1354113553.

Nave, C. (2018). J. Synchrotron Rad. 25, 1490-1504.

Schneider, G. (1998). Ultramicroscopy, 75, 85-104. 